\title{
Cyclosporine A at Reperfusion Reduces Infarct Size in Pigs
}

\author{
Andreas Skyschally • Rainer Schulz • Gerd Heusch
}

Published online: 25 February 2010

(C) The Author(s) 2010. This article is published with open access at Springerlink.com

Key words Cyclosporine A $\cdot$ Infarct size . Ischemia/reperfusion · Ischemic postconditioning . Myocardial infarction $\cdot$ Reperfusion injury

Timely reperfusion is mandatory for salvage of ischemic myocardium from irreversible damage. However, reperfusion induces damage per se, i.e. reperfusion injury contributes to final infarct size [1]. Ischemic postconditioning, i.e. brief episodes of intermittent coronary re-occlusion during early reperfusion, reduces infarct size. This protective effect was confirmed in all species tested so far [2], including humans [3], but common co-morbidities of ischemic heart disease may interfere with cardioprotective mechanisms including ischemic postconditioning [4].

The signal transduction of ischemic postconditioning is still unclear in detail [5]. Activation of "reperfusion injury salvage kinases" (RISK) is causal for ischemic postconditioning's protection in rodents [6]. In pigs, in which coronary anatomy and the spatial and temporal development of myocardial infarction are closer to that of humans, RISK activation is not mandatory for protection [7].

Supported by the German Research Foundation (He 1320/18-1).

A. Skyschally $\cdot$ R. Schulz $\cdot$ G. Heusch $(\bowtie)$

Institut für Pathophysiologie, Universitätsklinikum Essen,

Hufelandstraße 55,

45122 Essen, Germany

e-mail: gerd.heusch@uk-essen.de
The mitochondrial permeability transition pore (mPTP) is a potential end-effector of myocardial protection at reperfusion [8-10]. Cyclosporine A binds to cyclophilin D, inhibits $\mathrm{mPTP}$ opening and reduces infarct size $[9,11]$. Apart from experiments in rodents, cyclosporine A when given at reperfusion reduced infarct size in a proof-of-concept study in patients with acute myocardial infarction [12].

Protection by cyclosporine A at reperfusion was now tested in pigs. Enflurane-anesthetized Göttinger minipigs (20-40 kg body weight) of either sex were subjected to 90 min controlled hypoperfusion of the left anterior descending coronary artery and $120 \mathrm{~min}$ reperfusion [7]. In four pigs cyclosporine A $(5 \mathrm{mg} / \mathrm{kg}$ i.v. $)$ was infused 5 min before reperfusion; in four pigs, ischemic postconditioning was induced with six cycles of $20 \mathrm{~s}$ re-occlusion/ reperfusion each; four pigs with immediate full reperfusion served as controls. Systemic hemodynamics (Table 1) and subendocardial blood flow during ischemia (microspheres) were matched between groups (Fig. 1). Both, cyclosporine $\mathrm{A}$ at reperfusion and ischemic postconditioning reduced infarct size (TTC staining) to a similar extent compared to controls (Fig. 1).

Whereas pigs differ from rodents with respect to the causal role of RISK in ischemic postconditioning, they share with both rodents and importantly also humans protection by cyclosporine A at reperfusion, suggesting an important role for mitochondrial permeability transition pore opening across all species. 
Table 1 Systemic hemodynamics

\begin{tabular}{|c|c|c|c|c|c|c|}
\hline & & $\operatorname{HR}[1 / \mathrm{min}]$ & $\mathrm{LVP}_{\max }[\mathrm{mmHg}]$ & $\mathrm{dPdt}_{\max }[\mathrm{mmHg} / \mathrm{s}]$ & $\mathrm{CAP}_{\text {mean }}[\mathrm{mmHg}]$ & $\mathrm{CBF}_{\text {mean }}[\mathrm{ml} / \mathrm{min}]$ \\
\hline \multirow[t]{7}{*}{ Cyclosporine A } & Baseline & $99 \pm 7$ & $94 \pm 7$ & $1330 \pm 68$ & $112 \pm 5$ & $22.5 \pm 2.8$ \\
\hline & Isch5 & $100 \pm 6$ & $79 \pm 3$ & $959 \pm 55$ & $20 \pm 3 *$ & $1.9 \pm 0.1 *$ \\
\hline & Isch85 & $97 \pm 5$ & $77 \pm 6^{*}$ & $925 \pm 45$ & $20 \pm 2 *$ & $1.9 \pm 0.1^{*}$ \\
\hline & Rep 10 & $91 \pm 4$ & $68 \pm 7 *$ & $796 \pm 108$ & $102 \pm 4$ & $66.1 \pm 8.9 *$ \\
\hline & Rep30 & $108 \pm 7$ & $72 \pm 8^{*}$ & $1060 \pm 203$ & $105 \pm 1$ & $61.4 \pm 10.9^{*}$ \\
\hline & Rep60 & $115 \pm 7$ & $70 \pm 6^{*}$ & $946 \pm 117$ & $105 \pm 3$ & $54.8 \pm 7.2 *$ \\
\hline & Rep120 & $119 \pm 10$ & $65 \pm 7 *$ & $946 \pm 111$ & $103 \pm 5$ & $54.1 \pm 7.7 *$ \\
\hline \multirow[t]{7}{*}{ Postconditioning } & Baseline & $100 \pm 9$ & $96 \pm 3$ & $1446 \pm 36$ & $122 \pm 6$ & $24.6 \pm 3.1$ \\
\hline & Isch5 & $112 \pm 11$ & $77 \pm 7 *$ & $997 \pm 59$ & $22 \pm 2^{*}$ & $2.9 \pm 0.6^{*}$ \\
\hline & Isch85 & $105 \pm 9$ & $77 \pm 3^{*}$ & $1112 \pm 46$ & $24 \pm 2 *$ & $2.9 \pm 0.6^{*}$ \\
\hline & Rep10 & $109 \pm 8$ & $76 \pm 5^{*}$ & $1383 \pm 103$ & $109 \pm 6$ & $74.2 \pm 9.3 *$ \\
\hline & Rep30 & $113 \pm 8$ & $76 \pm 3^{*}$ & $1377 \pm 79$ & $111 \pm 4$ & $66.1 \pm 8.0^{*}$ \\
\hline & Rep60 & $113 \pm 11$ & $69 \pm 12^{*}$ & $1380 \pm 416$ & $122 \pm 4^{\#}$ & $65.8 \pm 10.9^{*}$ \\
\hline & Rep120 & $125 \pm 12$ & $69 \pm 5^{*}$ & $1364 \pm 259$ & $115 \pm 7$ & $59.1 \pm 14.7^{*}$ \\
\hline \multirow[t]{7}{*}{ Immediate full reperfusion } & Baseline & $93 \pm 6$ & $103 \pm 2$ & $1294 \pm 52$ & $122 \pm 5$ & $24.3 \pm 2.6$ \\
\hline & Isch5 & $98 \pm 10$ & $82 \pm 2 *$ & $1039 \pm 87$ & $23 \pm 2 *$ & $2.8 \pm 0.4^{*}$ \\
\hline & Isch85 & $94 \pm 7$ & $85 \pm 4^{*}$ & $1178 \pm 45$ & $23 \pm 1^{*}$ & $2.8 \pm 0.4^{*}$ \\
\hline & Rep10 & $95 \pm 7$ & $76 \pm 5^{*}$ & $1101 \pm 173$ & $111 \pm 7$ & $57.6 \pm 9.0 *$ \\
\hline & Rep30 & $103 \pm 12$ & $72 \pm 6^{*}$ & $1278 \pm 183$ & $106 \pm 4 *$ & $53.5 \pm 7.9 *$ \\
\hline & Rep60 & $118 \pm 16$ & $73 \pm 2 *$ & $1432 \pm 111$ & $110 \pm 1$ & $55.1 \pm 9.4 *$ \\
\hline & Rep120 & $116 \pm 15$ & $61 \pm 3^{*}$ & $1222 \pm 174$ & $118 \pm 9^{\#}$ & $67.1 \pm 16.8^{*}$ \\
\hline
\end{tabular}

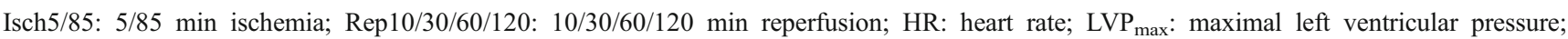
$\mathrm{dPdt}_{\max }$ : maximum in the first derivative of LVP; $\mathrm{CAP}_{\text {mean }}$ : mean coronary arterial pressure; $\mathrm{CBF}_{\text {mean }}$ : mean coronary blood flow; means $\pm \mathrm{SEM}$; ${ }^{*} p<0.05$ vs. Baseline; ${ }^{\#} p<0.05$ vs. Cyclosporine A; two-way-ANOVA with Fisher's LSD post-hoc tests.

Fig. 1 Infarct size with cyclosporine A given at reperfusion, postconditioning, and immediate full reperfusion; means \pm SEM; AAR: area at risk; ANOVA with Fisher's LSD post-hoc tests

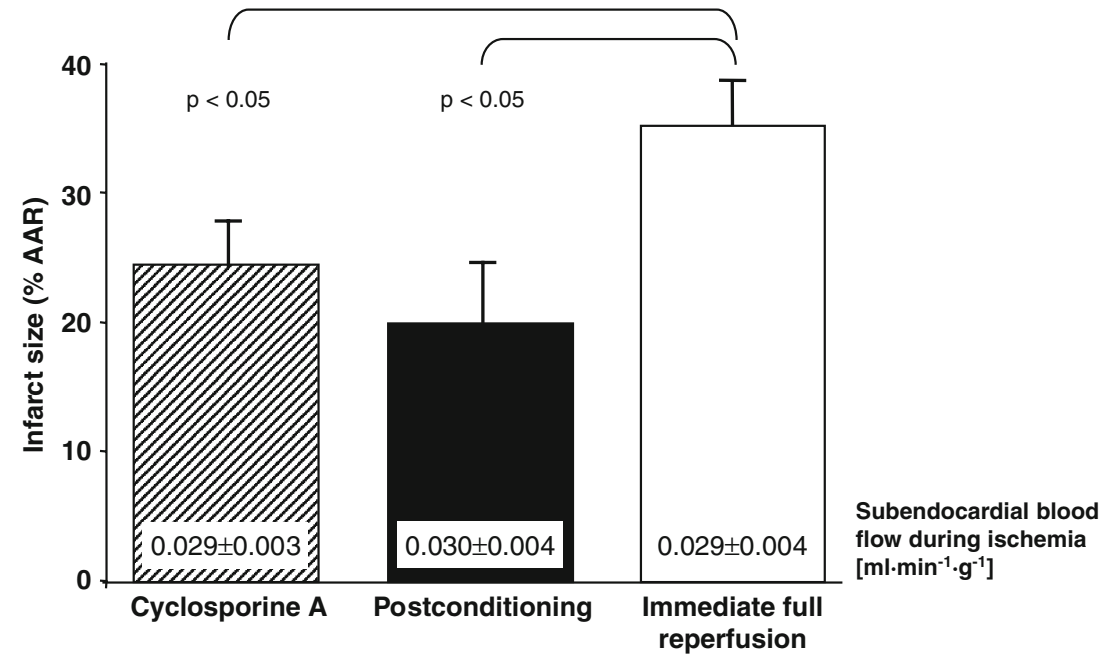


Open Access This article is distributed under the terms of the Creative Commons Attribution Noncommercial License which permits any noncommercial use, distribution, and reproduction in any medium, provided the original author(s) and source are credited.

\section{References}

1. Heusch G. Postconditioning. Old wine in a new bottle? J Am Coll Cardiol. 2004;44:1111-2.

2. Skyschally A, van Caster P, Iliodromitis EK, Schulz R, Kremastinos DT, Heusch G. Ischemic postconditioningexperimental models and protocol algorithms. Basic Res Cardiol. 2009;104:469-83.

3. Staat P, Rioufol G, Piot C, Cottin Y, Cung TT, L'Huillier I, et al. Postconditioning the human heart. Circulation. 2005;112:2143-8.

4. Ferdinandy P, Schulz R, Baxter GF. Interaction of cardiovascular risk factors with myocardial ischemia/reperfusion injury, preconditioning, and postconditioning. Pharmacol Rev. 2007;59:418-58.

5. Heusch G, Boengler K, Schulz R. Cardioprotection: nitric oxide, protein kinases, and mitochondria. Circulation. 2008;118:1915-9.
6. Hausenloy DJ, Yellon DM. Reperfusion injury salvage kinase signalling: taking a RISK for cardioprotection. Heart Fail Rev. 2007;12:217-34.

7. Skyschally A, van Caster P, Boengler K, Gres P, Musiolik J, Schilawa $\mathrm{D}$, et al. Ischemic postconditioning in pigs: no causal role for RISK activation. Circ Res. 2009;104:15-8.

8. Argaud L, Gateau-Roesch O, Raisky O, Loufouat J, Robert D, Ovize M. Postconditioning inhibits mitochondrial permeability transition. Circulation. 2005;111:194-7.

9. Lim SY, Davidson SM, Hausenloy DJ, Yellon DM. Preconditioning and postconditioning: the essential role of the mitochondrial permeability transition pore. Cardiovasc Res. 2007;75:530-5.

10. Heusch G, Boengler K, Schulz R. Inhibition of mitochondrial permeability transition pore opening: the holy grail of cardioprotection. Basic Res Cardiol. 2010;105:151-4.

11. Argaud L, Gateau-Roesch O, Muntean D, Chalabreysse L, Loufouat J, Robert D, et al. Specific inhibition of the mitochondrial permeability transition prevents lethal reperfusion injury. $\mathrm{J}$ Mol Cell Cardiol. 2005;38:367-74.

12. Piot C, Croisille P, Staat P, Thibault H, Rioufol G, Mewton N, et al. Effect of cyclosporine on reperfusion injury in acute myocardial infarction. N Engl J Med. 2008;359:473-81. 